\title{
Self-Trapped Nonlinear Matter Waves in Periodic Potentials
}

\author{
Tristram J. Alexander, Elena A. Ostrovskaya, and Yuri S. Kivshar \\ Nonlinear Physics Centre and ARC Centre of Excellence for Quantum-Atom Optics, \\ Research School of Physical Sciences and Engineering, Australian National University, Canberra ACT 0200, Australia
}

(Received 19 August 2005; published 30 January 2006)

We demonstrate that the recent observation of nonlinear self-trapping of matter waves in onedimensional optical lattices [Th. Anker et al., Phys. Rev. Lett. 94, 020403 (2005)] can be associated with a novel type of broad nonlinear state existing in the gaps of the matter-wave band-gap spectrum. We find these self-trapped localized modes in one-, two-, and three-dimensional periodic potentials, and demonstrate that such novel gap states can be generated experimentally in any dimension.

Bose-Einstein condensates (BECs) loaded into optical lattices show a wide range of complex nonlinear dynamics and intriguing physical properties [1-3]. One of the most striking effects is the possibility of spatial localization due to an interplay between periodicity and nonlinearity, even when the interatomic interaction is repulsive. Such localized states have long been known in nonlinear optics as gap solitons [4] whose frequencies lie inside the gaps of the linear band-gap spectrum of a periodic structure. In atomic physics, the experimental observation of BEC gap solitons proved to be a challenge due to the requirement of low atom numbers and densities [5]. However, more recently, a seemingly different localized state with steep edges and a large number of atoms was observed [6], where the increasing nonlinearity of the BEC wave packet enabled the transition from the diffusive regime of condensate expansion in a deep one-dimensional optical lattice to the regime where the initial expansion stopped and the width remained finite. This effect has been attributed to the self-trapping mechanism of energy localization described by discrete tight-binding nonlinear models [7], with similar effects first predicted for attractive nonlinearities in a purely discrete system [8].

In this Letter, we reveal that the observations of Ref. [6] can be explained by the excitation of a new type of gap state which serves as a missing link between the two fundamental types of nonlinear states in the lattice, the spatially extended nonlinear Bloch waves $[9,10]$, and the spatially localized gap solitons [10-12]. These new states may be viewed as "truncated" nonlinear Bloch waves localized in the gaps of the linear Bloch-wave spectrum. In what follows, we refer to them as "gap waves." Although the gap waves can be excited only above a certain density threshold, they exist well beyond the tight-binding regime, and therefore represent an important generalization of the self-trapping concept. Moreover, we find these nonlinear states in one-, two-, and three-dimensional periodic potentials. We also demonstrate that these novel gap states can be generated experimentally in any dimension, and for arbitrarily large initial atom numbers, by nonadiabatic loading of the BEC into a stationary optical lattice.
We consider a BEC cloud loaded into an optical lattice potential and described, in the mean-field approach, by the nonlinear Gross-Pitaevskii equation,

$$
i \frac{\partial \Psi}{\partial t}=-\Delta_{\mathrm{D}} \Psi+V_{L}(\mathbf{r}) \Psi+|\Psi|^{2} \Psi,
$$

where $\Psi$ is the macroscopic wave function, $\Delta_{\mathrm{D}}$ is the Laplacian operator acting in one-, two-, or three- (1D, 2D, or 3D) dimensions, and $V_{L}(\mathbf{r})=V_{0}\left(\sin ^{2} x+\sin ^{2} y+\right.$ $\sin ^{2} z$ ) is a periodic potential (in this case of a 3D lattice). Our model equation is made dimensionless by using the characteristic scales of the lattice, length $a_{L}=d / \pi$, energy $E_{\text {rec }}=\hbar^{2} / 2 m a_{L}^{2}$, and time $\omega_{L}^{-1}=\hbar / E_{\text {rec }}$, where $d$ is the lattice period and $m$ the mass of the trapped atoms. The wave function is in units of $1 / \sqrt{8 \pi a_{L}^{2}\left|a_{s}\right|}$ where $a_{s}$ is the scattering length of the condensed atoms, which we take to be positive.

The stationary condensates in a lattice are described by solutions of Eq. (1) of the form: $\Psi(\mathbf{r}, t)=\psi(\mathbf{r}) \exp (-i \mu t)$, where $\mu$ is the corresponding chemical potential. When the interatomic interaction can be neglected, these states have the form of linear Bloch waves, $\psi(\mathbf{r})=\phi_{\mathbf{k}}(\mathbf{r}) \exp (i \mathbf{k r})$, where the wave vector $\mathbf{k}$ is selected in the Brillouin zone, and $\phi_{\mathbf{k}}(\mathbf{r})=\phi_{\mathbf{k}}(\mathbf{r}+\mathbf{d})$ is a periodic function with the periodicity of the lattice. The spectrum $\mu(\mathbf{k})$ of the atomic Bloch waves in the optical lattice has a band-gap structure.

For specific combinations of a trapping potential and an optical lattice, Eq. (1) lends itself to a dimensionality reduction procedure. For example, in the case of a cigarshaped BEC cloud trapped in a 1D optical lattice and tightly confined in the directions perpendicular to the lattice, the dimensionality reduction results in a $1 \mathrm{D}$ version of Eq. (1), with $V_{L}(\mathbf{r})=V_{0} \sin ^{2} x$, suitable for modeling the experimental situation of Anker et al. [6]. We consider different types of stationary solutions of this 1D reduction. The linear Bloch-wave spectrum $\mu(k)$ for the 1D lattice is shown in Fig. 1(a). In the presence of interaction, each linear Bloch wave will have an associated family of spatially extended stationary states, nonlinear Bloch waves, with a similar spatial structure, but with a maximum density dependent on the chemical potential $[9,10]$. The 

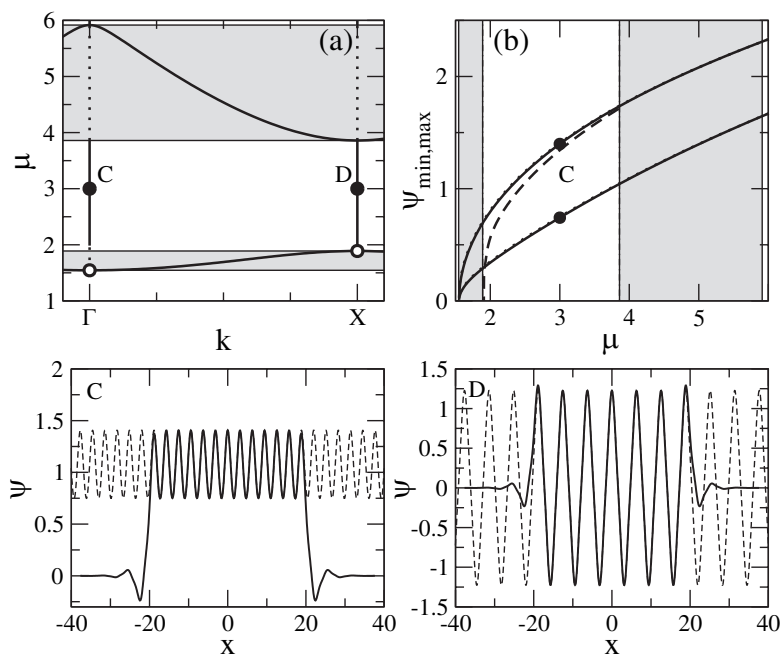

FIG. 1. (a) Band-gap diagram of the 1D optical lattice showing bifurcation of nonlinear Bloch waves (dotted lines) from the band edges (open circles) and the existence of gap waves (solid lines). Solid circles correspond to the gap waves C and D at bottom. Dashed lines at bottom are the associated nonlinear Bloch waves. (b) Maximum (upper line) and minimum (lower line) amplitudes of the ground-state nonlinear Bloch wave vs chemical potential and the approximate solution (dotted, overlying solid lines); gap soliton maximum (dashed lines); and linear bands (shaded). In all plots, $V_{0}=4$.

ground state of an interacting BEC in the lattice is therefore a nonlinear Bloch wave that transforms into the linear Bloch wave at the center of the Brillouin zone for vanishingly small atomic densities. As the density of this state increases, the chemical potential may actually shift into the linear band gap [see Fig. 1(b)]. The ground-state nonlinear Bloch wave [dashed line in Fig. 1(c)] has a nonzero minimum density, unlike the excited Bloch-wave states [Fig. 1(d), dashed].

Other types of stationary matter-wave states are localized in space. For the repulsive condensate, such states exist only in the gaps of the linear Bloch-wave spectrum, in the form of gap solitons; they have been discussed theoretically $[10,11]$ and recently demonstrated experimentally [5]. The peak density of the matter-wave gap solitons increases monotonically from the lower gap edge through the entire gap [see Fig. 1(b)]. Near the lower gap edge the soliton has the structure of a slowly varying envelope superimposed onto the corresponding Bloch state. The number of atoms in localized gap solitons is limited and can be very small [e.g., $\sim 250$ atoms in [5]], making it a challenge to observe them experimentally.

However, the optical lattice can support yet another, new type of localized state, which is not atom number limited. These are the so-called gap waves, which are, in their essence, truncated nonlinear Bloch waves. These states exist when the chemical potential of a nonlinear Bloch wave is shifted into the linear gap. Figure 1 (bottom) shows two examples of these gap waves and their relationship with the associated (nontruncated) nonlinear Bloch waves. Unlike the conventional gap solitons, the gap waves can be associated with a nonlinear Bloch state corresponding to any band edge (see Fig. 1), and not only the one at the lower gap edge. Below we consider the gap waves associated with the ground-state nonlinear Bloch wave.

The truncation of nonlinear Bloch waves, and therefore the existence of gap waves, is inherently linked to the gap solitons. A gap soliton guides us in estimating the maximum density which can be localized due to Bragg reflection. We can expect that if, at a given chemical potential inside the gap, the minimum density of a nonlinear Bloch wave $\left|\psi_{\text {Bloch,min }}(\mu)\right|$ is larger than the peak density of a gap soliton $\left|\psi_{\text {sol,max }}(\mu)\right|$, the nonlinear repulsive effects will outweigh the localization effects due to periodicity, and there will be no associated gap-wave state. With this in mind the condition $\left|\psi_{\text {Bloch, min }}(\mu)\right|=\left|\psi_{\text {sol,max }}(\mu)\right|$ defines the boundary of the existence region of the ground-state gap wave, i.e., the truncated nonlinear ground-state Bloch wave. Figure 2 (dashed line) shows a good agreement between the existence boundary found using this condition and the full numerical calculation. The existence domain will always begin at some finite detuning of the chemical potential from the gap edge, because the peak density of a gap soliton is zero at the gap edge whereas the minimum density of the ground-state nonlinear Bloch wave is finite [see the comparison in Fig. 1(b)]. As the lattice becomes weaker the existence region becomes smaller, until finally reaching a cutoff at the upper gap edge [see Fig. 2] for the
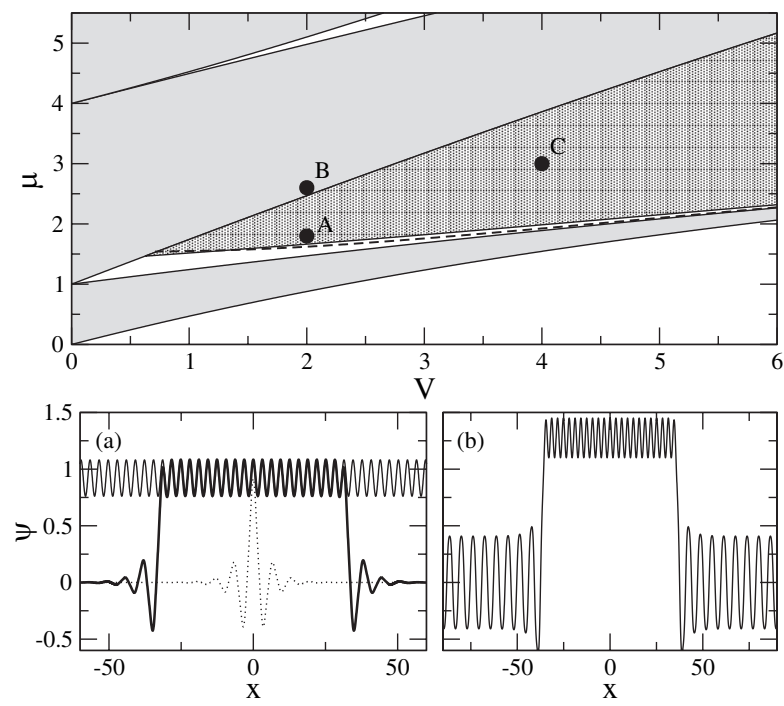

FIG. 2. Top: existence region of ground-state gap waves (dark shading) inside the first linear band gap, for varying $V_{0}$. Closed circles correspond to the examples at bottom [(a), (b)] or in Fig. 1(c). Dashed line shows the calculated boundary (see text). Below: (a), a gap wave near cutoff (thick solid line) compared to a gap soliton (dotted line) and the approximate solution for the nonlinear Bloch wave (thin solid line) $\left(V_{0}=2, \mu=1.8\right)$; (b), a gap wave moved to the band $\left(V_{0}=2, \mu=2.6\right)$. 
1D lattice depth of $V_{0}=0.63$, far from the tight-binding regime.

The form of the ground-state nonlinear Bloch wave may be modeled accurately using the trial function $\Psi(x)=$ $C_{1}+C_{2} \cos (2 x)$, where $C_{1}$ and $C_{2}$ are determined by substituting $\Psi(x)$ into the full system Hamiltonian $H$ and solving the variational problem $\delta H / \delta C_{1}=\delta H / \delta C_{2}=0$. The coefficients may then be found from the algebraic equations,

$$
\begin{aligned}
4 C_{1}^{3}-V_{0} C_{2}+2 C_{1}\left(3 C_{2}^{2}-2 \mu+V_{0}\right) & =0, \\
3 C_{2}^{3}-2 V_{0} C_{1}+2 C_{2}\left(8+6 C_{1}^{2}-2 \mu+V_{0}\right) & =0 .
\end{aligned}
$$

In Fig. 1(b) we see that the approximate solution for the Bloch-wave amplitudes cannot be distinguished from the numerical one. The shape of the gap waves is also in very good agreement with the approximate solution [see Fig. 2 (bottom)], which provides further confirmation of the nonlinear Bloch-wave origin of these localized states.

The gap waves described above closely resemble the nonlinearly self-trapped states of the BEC in a onedimensional lattice observed in the recent experiment [6]. The latter are characterized by steep edges of the density distribution that are formed after the initial expansion of the atomic cloud. These experimental observations were made in a deep optical lattice $\left(V_{0} \sim 11\right)$ with approximately 5000 atoms. Here we demonstrate that the resulting dynamics is general, provided certain critical conditions are met, and more importantly may also be observed with more shallow optical lattices and larger atom numbers. In order to simulate the generation process, we use as an initial state a spatially localized BEC cloud trapped in a parabolic potential and well described by the ThomasFermi (TF) approximation. We release this cloud into the $1 \mathrm{D}$ optical lattice nonadiabatically at $t=0$, and then study the BEC evolution in the lattice for varying particle number.

Figure 3 shows a typical example of the resulting dynamics. The initial TF distribution evolves toward the ground-state nonlinear Bloch mode of the lattice. The finite extent of the input ensures that the nonlinear Bloch wave must be truncated. Localization then occurs through Bragg reflection, with characteristic sharp boundaries. Excitations reflected from these boundaries lead to running waves moving across the localized state which slowly decay over time due to radiation [see Figs. 3(b) and 3(c)]. After a sufficient evolution the excitations are damped and the generated state closely resembles a ground-state gap wave, as shown in Fig. 3(d).

The condition for the successful generation of a gap wave may be estimated by comparing the initial TF distribution with the target gap state. The maximum gap wave which could possibly be generated is that with the same number of particles as the initial state, and the same spatial extent. However, as we see in evolution, an approximate $30 \%$ atom loss occurs through radiation of atoms into a low
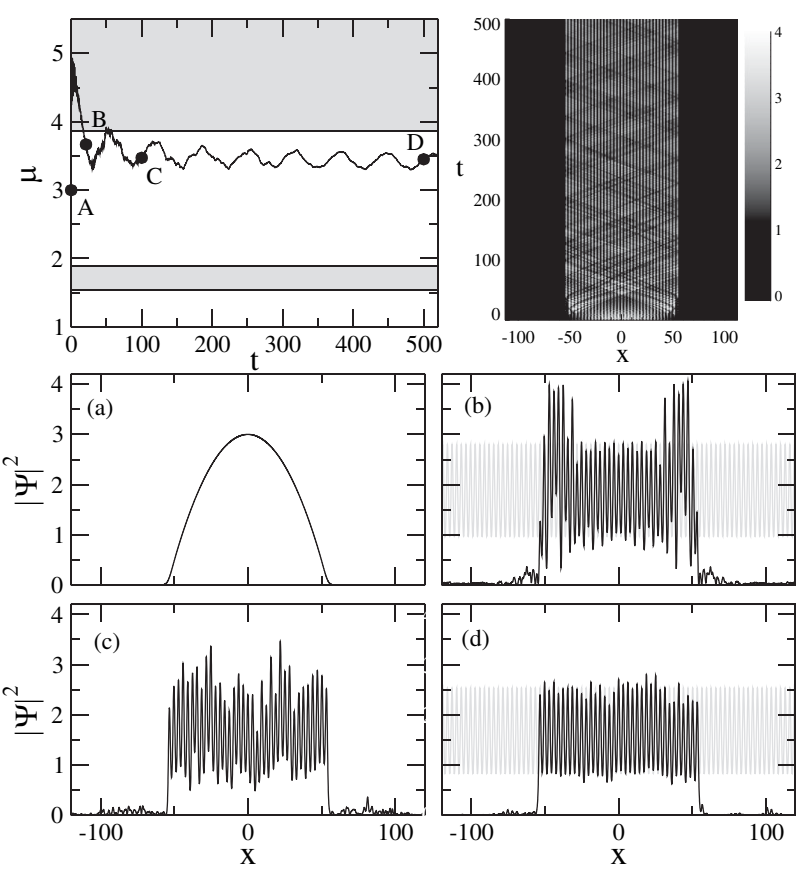

FIG. 3. Dynamical generation of a gap wave from a ThomasFermi state released into an optical lattice $\left(V_{0}=4\right)$. Top: left, evolution of the chemical potential; right, density plot showing reflections of excitations from steep boundaries. Lower plots show snapshots at points labeled in top demonstrating convergence to a gap wave. Light colored lines [in (b) and (d)] show the nonlinear Bloch wave at that chemical potential.

density background. Using the variational solution of Eq. (2) to calculate the gap-wave atom number, and allowing for the atom loss, we find that for a given gap wave with coefficients $\left(C_{1}, C_{2}\right)$ the initial TF profile must have a peak density of $2 C_{1}^{2}+C_{2}^{2}\left(=\mu_{\mathrm{TF}}\right)$. This is a minimum condition on the density. Higher density initial conditions may also be used, even to the extent that they generate a state in the band (see Fig. 2) and then radiate away particles until they descend into the gap and are localized.

To link these results to experiment we note that the normalized density is multiplied by $10^{-6} \sqrt{2} /\left(8 \pi a_{L}^{2}\left|a_{s}\right|\right)$ to give the peak physical density $n_{\text {peak }}$ in atoms $\mathrm{cm}^{-3}$. For ${ }^{87} \mathrm{Rb}$ in a $1 \mathrm{D}$ optical lattice with period $2 \mu \mathrm{m}$ and a trapping frequency of $\omega_{\perp}=2 \pi \times 450 \mathrm{~Hz}$ the initial condition of Fig. 3 has a maximum density of $8.3 \times 10^{13}$ atoms $\mathrm{cm}^{-3}$ and total atom number of 8200 . The generated gap wave contains 6400 atoms with approximately 180 atoms per well. In [6] the lower density in the deep lattice actually places the gap wave near the existence cutoff. To avoid transverse excitations, and therefore remain in the quasi-1D regime, the constraint $n_{\text {peak }} a_{s} \pi \hbar / m \omega_{\perp} \ll 1$ must be satisfied [13]. The maximum allowable peak density may therefore be increased by increasing the transverse trapping frequency. We note here that in the tightbinding analysis of the experiment [6] it was assumed that transverse excitations did indeed play a role, leading to an 

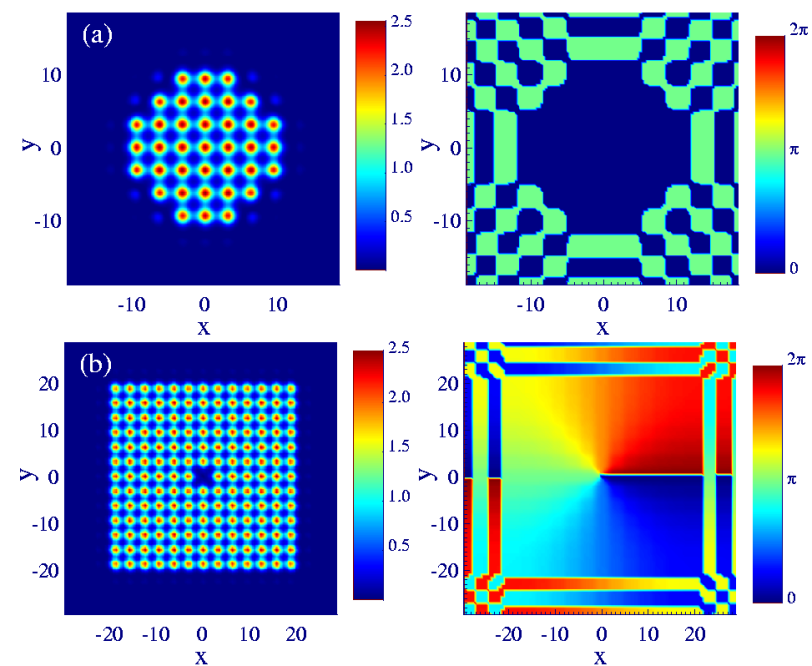

FIG. 4 (color online). Examples of gap waves in a 2D lattice associated with the ground-state nonlinear Bloch wave $\left(V_{0}=4\right.$, $\mu=4.5$ ) showing density (left) and phase (right). (a) Broad nonsquare mode, (b) square mode with imprinted vortex.

effective interaction term proportional to $|\psi|$ and not to $|\psi|^{2}[6,14]$; however, as we show here the resulting selftrapping dynamics is general to potentials far from the tight-binding limit.

Relaxing the dimensionality constraint we demonstrate that gap waves may also exist in 2D and 3D lattices. In Fig. 4(a) we show an example of a 2D gap wave associated with the ground-state nonlinear Bloch wave, connected to the linear Bloch wave at the center of the Brillouin zone. Remarkably, this gap wave can be used as a background that supports nontrivial phase states formed by phase imprinting, and provides a means for obtaining a dynamically stable stationary vortex [see Fig. 4(b)], which is related to the broad vortex states spatially localized inside the $2 \mathrm{D}$ gap [15].

Similarly, we find that the gap waves exist in fully 3D lattices, for a wide range of the system parameters, with an example shown in Fig. 5(a). The cross-section of the condensate wave function [Fig. 5(b)] shows that it is indeed connected to the ground-state nonlinear Bloch wave. As with the 1D geometry discussed above, generation of a specific 3D gap wave is best done nonadiabatically. Figures 5(c) and 5(d) show the generation of the 3D broad gap wave after an initially spherically symmetric trapped condensate is released into a 3D optical lattice. Similar to the 1D case, to observe these dynamics for a particular lattice depth, the initial condensate density must be sufficient to excite the gap wave.

In conclusion, we have demonstrated that the recent experimental observation of the nonlinear self-trapping effect in one-dimensional optical lattices can be associated with the existence of a new type of localized gap state. We have revealed that these robust nonlinear modes exist in all
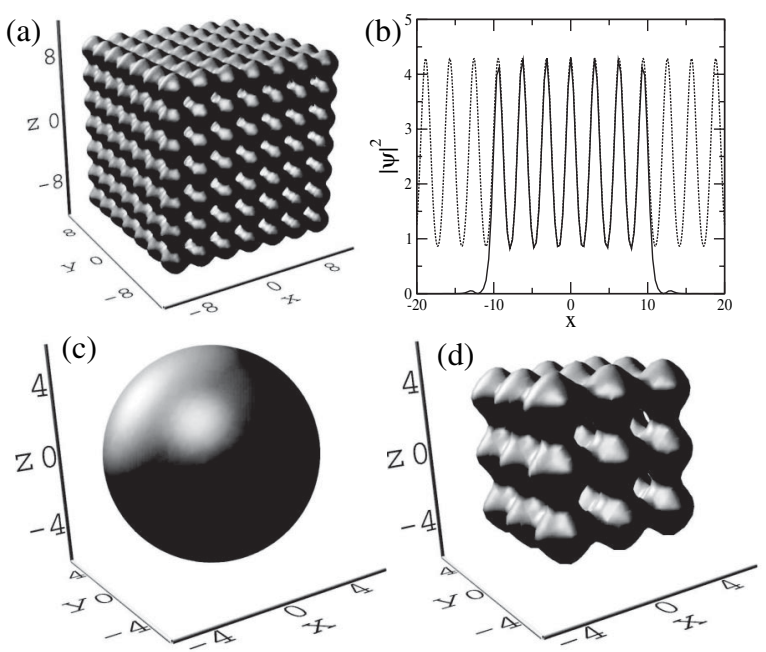

FIG. 5. (a) Constant density surface of a $3 \mathrm{D}$ gap wave at $V_{0}=$ 6 and $\mu=7.9$. (b) Density cut through (a) and nonlinear Bloch wave (dashed line). (c), (d) Generation of 3D gap wave from Thomas-Fermi solution, which in physical units for ${ }^{87} \mathrm{Rb}$ in a lattice with a $2 \mu \mathrm{m}$ period corresponds to (c) input, maximum density $5.9 \times 10^{13}$ atoms cm $\mathrm{cm}^{-3}$ (6450 atoms) and (d) gap wave at $t=50(\sim 50 \mathrm{~ms})$, with maximum density $1.1 \times 10^{14}$ atoms $\mathrm{cm}^{-3}$ (4000 atoms).

dimensions, and they can have arbitrary extension within the lattice. We have demonstrated that these states provide an important missing link between earlier described gap solitons and nonlinear Bloch waves, and we have identified the key requirements for their generation.

The authors acknowledge useful discussions with S. Darmanyan, S. Flach, and R. Hulet and the support of the Australian Research Council (ARC).

[1] B. Eiermann et al., Phys. Rev. Lett. 91, 060402 (2003).

[2] L. Fallani et al., Phys. Rev. Lett. 91, 240405 (2003).

[3] M. Modugno et al., Phys. Rev. A 70, 043625 (2004).

[4] Yu.S. Kivshar and G. P. Agrawal, Optical Solitons: From Fibers to Photonic Crystals (Academic, New York, 2003).

[5] B. Eiermann et al., Phys. Rev. Lett. 92, 230401 (2004).

[6] Th. Anker et al., Phys. Rev. Lett. 94, 020403 (2005).

[7] A. Trombettoni and A. Smerzi, Phys. Rev. Lett. 86, 2353 (2001).

[8] S. Darmanyan et al., Phys. Rev. B 59, 5994 (1999).

[9] J. C. Bronski et al., Phys. Rev. Lett. 86, 1402 (2001).

[10] P. J. Louis et al., Phys. Rev. A 67, 013602 (2003).

[11] O. Zobay et al., Phys. Rev. A 59, 643 (1999).

[12] V. Ahufinger et al., Phys. Rev. A 69, 053604 (2004).

[13] L. Pitaevskii and S. Stringari, Bose-Einstein Condensation (Oxford, New York, 2003), Section 17.2.

[14] A. Smerzi and A. Trombettoni, Phys. Rev. A 68, 023613 (2003).

[15] E. A. Ostrovskaya and Yu. S. Kivshar, Phys. Rev. Lett. 93, 160405 (2004). 